\title{
Laser accelerated protons captured and transported by a pulse power solenoid
}

\author{
T. Burris-Mog, ${ }^{1}$ K. Harres, ${ }^{2}$ F. Nürnberg, ${ }^{2}$ S. Busold, ${ }^{2}$ M. Bussmann, ${ }^{1}$ O. Deppert, ${ }^{2}$ G. Hoffmeister, ${ }^{2}$ M. Joost, ${ }^{1}$ \\ M. Sobiella, ${ }^{1}$ A. Tauschwitz, ${ }^{3}$ B. Zielbauer, ${ }^{3,4}$ V. Bagnoud,${ }^{3}$ T. Herrmannsdoerfer, ${ }^{1}$ M. Roth, ${ }^{2}$ and T. E. Cowan ${ }^{1}$ \\ ${ }^{1}$ Helmholtz-Zentrum Dresden-Rossendorf (HZDR), Bautzner Landstraße 400, D-01328 Dresden, Germany \\ ${ }^{2}$ Technische Universität Darmstadt (TUD), Schlogartenstraße 9, D-64289 Darmstadt, Germany \\ ${ }^{3}$ GSI Helmholtz-Zentrum für Schwerionenforschung GmbH (GSI), Planckstraße 1, D-64291 Darmstadt, Germany \\ ${ }^{4}$ Helmholtz-Institute Jena (HIJ), Helmholtzweg 4, D-07743 Jena, Germany
}

(Received 15 August 2011; published 7 December 2011)

\begin{abstract}
Using a pulse power solenoid, we demonstrate efficient capture of laser accelerated proton beams and the ability to control their large divergence angles and broad energy range. Simulations using measured data for the input parameters give inference into the phase-space and transport efficiencies of the captured proton beams. We conclude with results from a feasibility study of a pulse power compact achromatic gantry concept. Using a scaled target normal sheath acceleration spectrum, we present simulation results of the available spectrum after transport through the gantry.
\end{abstract}

DOI: 10.1103/PhysRevSTAB.14.121301

PACS numbers: 41.75.Jv, 87.55.- $\mathrm{x}, 87.56 . \mathrm{bd}$

Over the past decade, there has been extensive research regarding the acceleration of protons driven by intense laser light $\left(I>10^{18} \mathrm{~W} / \mathrm{cm}^{2}\right)$. The laser interacts with solid foil targets which leads to the acceleration mechanism known as target normal sheath acceleration (TNSA) [1]. TNSA produces an electrostatic acceleration field of the order $\mathrm{TV} / \mathrm{m}$ on the rear side of the foil while the foil's contaminant layer of $\mathrm{H}_{2} \mathrm{O}$ and hydrocarbons provides the source of protons as well as oxygen and carbon ions. Although laser accelerated proton beams are broadband and possess large divergences, their pursuit is driven by their high yields (up to $10^{13}$ protons per laser pulse) and their ultralow emittances (transversely 100-fold better and longitudinally at least $10^{4}$-fold better than conventional accelerators [2]). Possible applications may include a hybrid system that combines a laser accelerated source with a conventional postaccelerator [2-6] or a distantfuture laser accelerator with a compact and cost effective beam transport system for cancer therapy [2,6-9]. Because TNSA results in a large diverging proton beam, both applications will require efficient capture and collimation by a focusing element such as a magnetic lens.

The coupling of magnetic lenses with laser accelerators has previously been accomplished with permanent magnet quadrupoles (PMQs) where the capture of electrons [10] and protons [11,12] were demonstrated. Although PMQs are widely used in conventional accelerator beam lines to correct paraxial beams with small divergences, their magnetic field is on the order of $1 \mathrm{~T}$ and therefore cannot sufficiently capture highly divergent protons of more than

Published by the American Physical Society under the terms of the Creative Commons Attribution 3.0 License. Further distribution of this work must maintain attribution to the author(s) and the published article's title, journal citation, and DOI. a few $\mathrm{MeV}$, let alone the $250 \mathrm{MeV}$ protons necessary for proton therapy. DC resistive magnets are limited by Ohmic heating, and the more effective DC superconducting magnets are limited by magnetic quenching. Because laser accelerated protons are accelerated on the picosecond time scale, one can take advantage of pulse power technology. In this work, a pulse power solenoid was used to generate temporally short but intense magnetic fields. As protons enter the radial fringe fields of the solenoid, the Lorentz force $\mathbf{F}=q \mathbf{v} \times \mathbf{B}$ causes an azimuthal acceleration. The resulting azimuthal velocity $v_{\phi}$ leads to an inward radial force when it interacts with the longitudinal magnetic field $B_{z}$ within the bore. Compared to quadrupoles, a solenoid lens can accommodate larger acceptance angles and larger transport efficiencies. Moreover, the magnetic field strength $B$ needed when scaling to higher proton energies $E_{p}$ scales as $B \propto E_{p}{ }^{1 / 2}$, and therefore up to $250 \mathrm{MeV}$ protons can be accommodated with the technology discussed in this article.

The work presented here demonstrates the effectiveness of proton capture and transport by a solenoid pulsed at two different magnetic field strengths, 7.2 and 8.5 T. This pulse power solenoid is a first-generation design and, based on our results, later designs will be optimized. As illustrated in

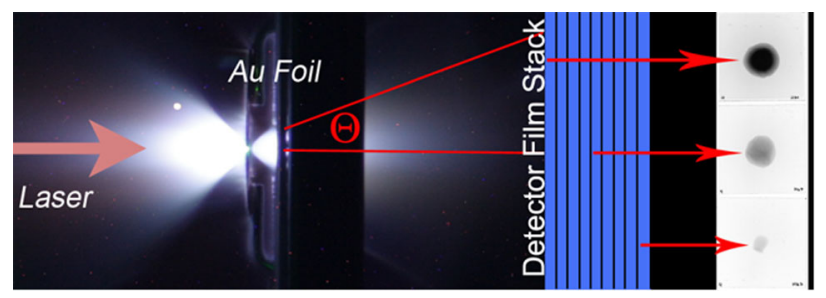

FIG. 1. Dosimetric film stack illustrating the recording of laser accelerated protons and the decrease in divergence angles $\Theta$ for more energetic protons. 


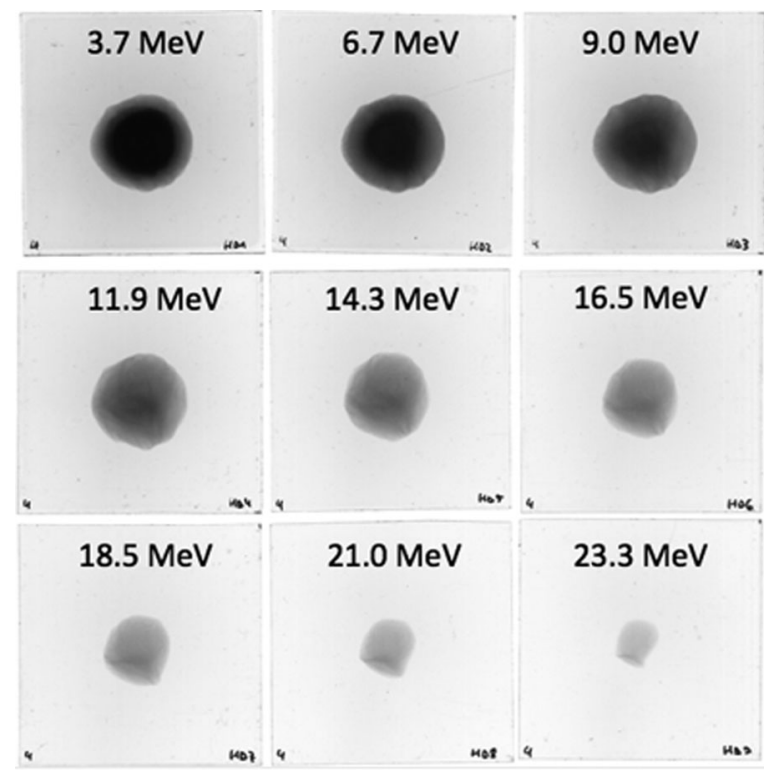

FIG. 2. Direct measurement of laser accelerated protons from a single PHELIX laser pulse. The low energy protons deposit a larger dose and have a larger beam profile than the higher energy protons.

Fig. 1, the energy-resolved and spatially resolved proton spectra were recorded with a layered dosimetric film stack of radiochromic films [13]. The proton beam spectrum and the energy-dependent angular envelopes for the presented setup were extracted [14] from the film (Fig. 2) and used to generate a simulated laser accelerated proton beam. Using simulation with measured data as the input parameters allows one to infer the transport efficiencies of the particles that pass through the solenoid. Tracking of the simulated protons was performed with the computer code GENERAL PARTICLE TRACER (GPT) version 3.01 [15], and postprocessing routines were developed to recreate the dosimetric films irradiated during the experiment. We briefly present a concept design for a therapeutic pulse power proton gantry with the presented solenoid acting as the front-end capture element. A more detailed description of the gantry will follow in a later publication.

\section{EXPERIMENTAL SETUP}

The experiment was performed at the PHELIX laser system (petawatt high energy laser for ion experiments [16]) at the GSI-Helmholtz-Zentrum für Schwerionenforschung $\mathrm{GmbH}$ in Darmstadt, Germany. Intensities of $2.9 \times 10^{19} \mathrm{~W} / \mathrm{cm}^{2}$ were produced with $72 \mathrm{~J}$ of normal incident linearly polarized $1.054 \mu \mathrm{m}$ laser light in $500 \mathrm{fs}$. The laser light was focused to an $8.5 \mu \mathrm{m}$ by $17 \mu \mathrm{m}$ diameter spot size (FWHM) on a flat $25 \mu \mathrm{m}$ thick $\mathrm{Au}$ foil, and the spot size contained $22 \%$ of the laser energy. The proton beams accelerated by the PHELIX laser system are contained within a relatively uniform angular distribution with up to a half-angle divergence of $7^{\circ}$ for high energy protons and $20^{\circ}$ for low energy protons (Fig. 2). The acceleration process completes on the picosecond time scale, and the exponentially decaying proton spectra for this experiment had a maximum cutoff near $23 \mathrm{MeV}$. A $46 \mu \mathrm{m} \mathrm{Cu}$ foil was placed in front of each film layer within the dosimetric film stack, and the first $\mathrm{Cu}$ foil stopped all protons with energies less than $3.7 \mathrm{MeV}$ resulting in a low energy cutoff of the spectrum (Fig. 3, black solid line, top and right axes). Integration over the experimental spectrum shows that $2 \times 10^{12}$ protons with energies greater than 3.7 MeV were accelerated from the rear of the foil. A conservative approach was used to infer a scaled TNSA spectrum with a maximum proton energy of $250 \mathrm{MeV}$ (Fig. 3, red dashed line, bottom and left axes). We assume that the total number of protons accelerated remains unchanged and that a larger magnitude electrostatic sheath increases each proton's energy by a factor of $250 \mathrm{MeV} / 23 \mathrm{MeV}=10.87$. This yields a reduction in $d N / d E$ per $\mathrm{MeV}$ and a maximum proton energy of $250 \mathrm{MeV}$.

The experimental setup (Fig. 4) shows the $300 \mu \mathrm{H}$ solenoid that was produced at the Helmholtz-Zentrum Dresden-Rossendorf in a collaboration between the Institute for Radiation Physics and the Dresden High Magnetic Field Laboratory. It has a $48 \mathrm{~mm}$ bore and is $150 \mathrm{~mm}$ in length, and a maximum tested on-axis field of $16 \mathrm{~T}$ has been repeatedly reached while preserving structural integrity. The front end of the solenoid (i.e. starting from the windings) was positioned $95 \mathrm{~mm}$ from the $\mathrm{Au}$
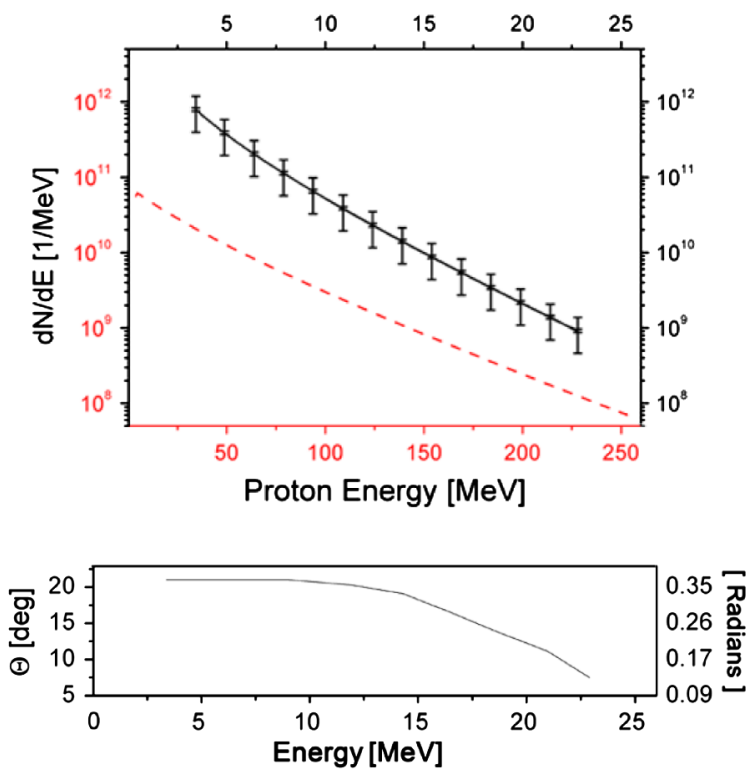

FIG. 3. Top: Processed results of the data presented in Fig. 2 (black solid curve, top and right axes, includes 5\% radiochromic film imaging spectroscopy error bars and 50\% shot-to-shot error bars) along with a scaled (predicted) TNSA spectrum with maximum energy of $250 \mathrm{MeV}$ (red dashed curve, bottom and left axes). Bottom: Maximum beam divergence as a function of proton energy. 


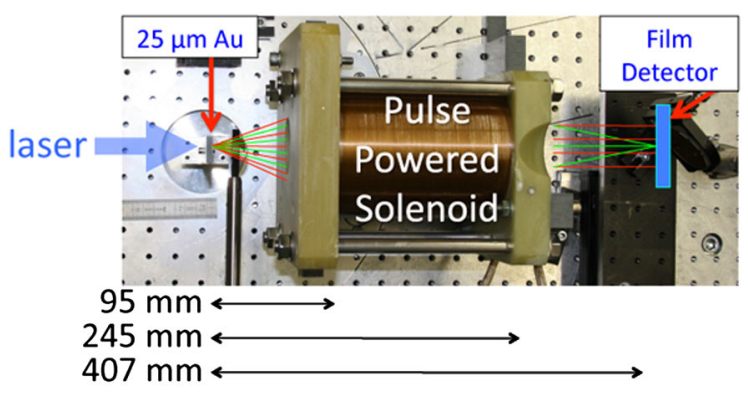

FIG. 4. Experimental setup: The windings of the solenoid begin $95 \mathrm{~mm}$ from the target. Laser-target alignment optics and dosimetric film are positioned under vacuum using motor stages.

target foil, and the solenoid was aligned on axis to within $1 \mathrm{~mm}$. The current in the solenoid reached a maximum after a $700 \mu$ s rise time, and a set of crowbar diodes critically damped the fall within $2 \mathrm{~ms}$ (Fig. 5). The changing magnetic field induces currents in nearby conductive materials, but if necessary, the resulting induced magnetic fields can be suppressed during the rise of the current through the use of an Eddy shield.

The radiochromic films in Fig. 6 show the spatial distribution of protons for magnetic field strengths of $7.2 \mathrm{~T}$ (top) and $8.5 \mathrm{~T}$ (bottom), and each layer of film is most sensitive to the Bragg-peak deposition of energy which also allows for an energy resolution. The film has a high reliability, a high spatial resolution, and is commonly used in medical dosimetry $[17,18]$. The basic features seen in Fig. 6 are 3.7 $\mathrm{MeV}$ protons diverging after being focused and, for the $8.5 \mathrm{~T}$ field, a near focus of $6.7 \mathrm{MeV}$ protons and a near collimation of $14.3 \mathrm{MeV}$ protons. Limb brightening can be seen and is a result of spherical aberrations. The striations within the early film layers are reproducible and evidence of additional perturbations which we have traced to imperfections in the magnetic field. This will be discussed

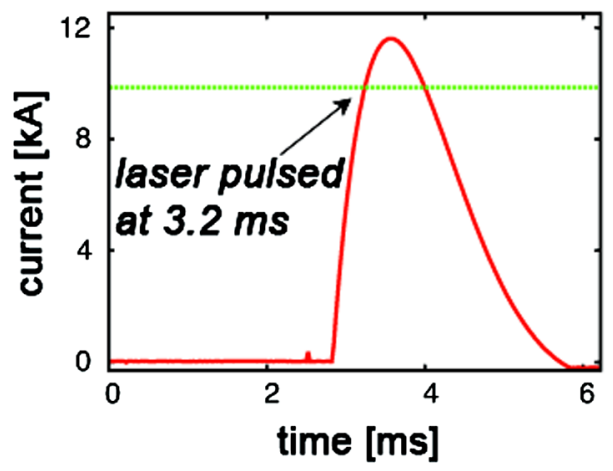

FIG. 5. Plot of measured current through the solenoid as a function of time. The solenoid is pulsed at time $t=2.85 \mathrm{~ms}$, and the laser is pulsed at $t=3.20 \mathrm{~ms}$. The current at the time of the laser pulse is $9.85 \mathrm{kA}$ as indicated by the green dashed line and can be considered static since the acceleration time is on the ps time scale and propagation time through the magnetic field is on the ns time scale.

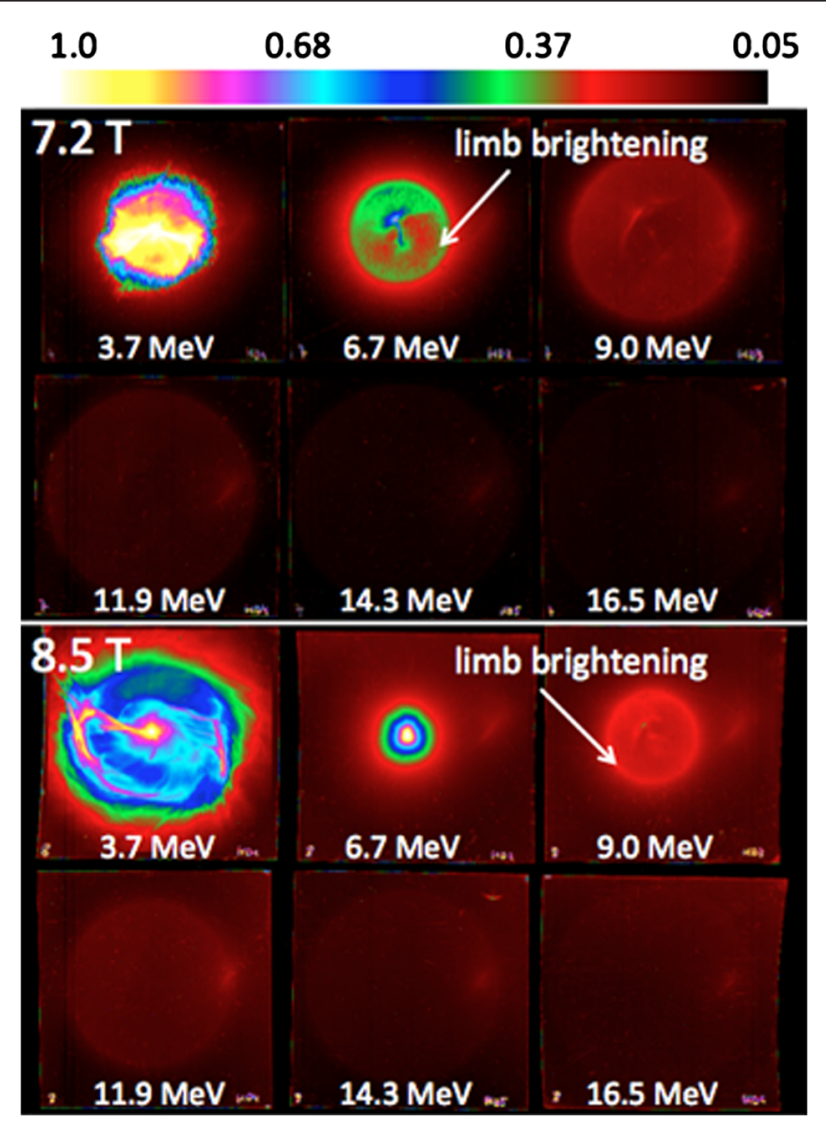

FIG. 6. Radiochromic film images: The films record the transverse position of the protons after they have been transported through a 7.2 T (top) and 8.5 T (bottom) solenoidal field. Proton energies corresponding to Bragg peak dose depositions are shown at the bottom of each film, and arrows point to areas of limb brightening.

later. For a quantitative understanding of the film, including proton yields and aberrations, we have performed computer simulations using measured data as the input parameters.

\section{SIMULATION}

The GPT computer code was developed for conventional accelerators and beam transport systems, and the superpositioning of magnetic fields and the ability to track charges through those fields are well established and applicable for our intended use. Although the solenoid's magnetic field has a rise time of $\sim 1 \mathrm{~ms}$, the magnetic field of the solenoid can be considered constant during the few nanoseconds needed for the protons to travel through the field.

The simulated source was generated via the Monte Carlo method. The input parameters were taken from direct measurements of the laser accelerated protons and include the energy-dependent half angles through the use of a von Mises [19] distribution. The source contained $1 \times 10^{6}$ simulated protons. The simulated energy deposition and 


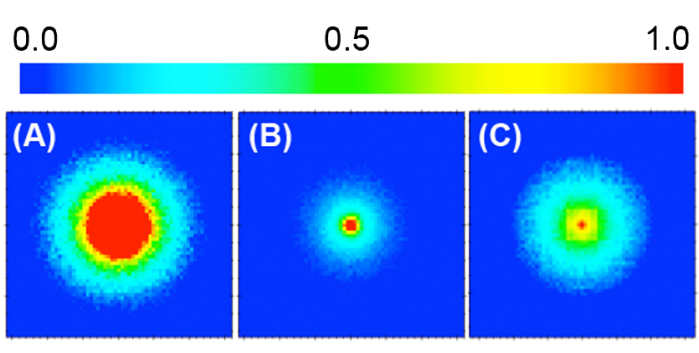

FIG. 7. Illustration of the generation of film response to protons from simulated data. (A) Proton density in a simulated layer of film. (B) Dose deposited from the protons in the left image. (C) Final result after weighting the deposited dose with the optical density response of the film.

optical response of the simulated dosimetric film was corrected to represent $2 \times 10^{12}$ protons in total. The copropagating electrons associated with TNSA have been excluded. Previous experiments [2] demonstrate that the stripping of the copropagating electrons from the plasma expansion does not significantly affect the proton emittance. Preliminary simulation results [20] of electrons copropagating with protons through a solenoid, however, do indicate a small electric field lensing effect acting mostly on the protons near the axis. In the presented simulation, space charge effects are excluded, and this remains an area of future study.

Transport efficiencies along with spherical, chromatic, and higher order aberrations are characterized by tracking the simulated protons and recording their trajectories. To model the radiochromic film, a postprocessing routine was developed to convert the simulated protons' positions and momentums at the film into the optical response of the film, and the process is as follows. The protons' positions and momentums at the film layer were recorded [Fig. 7(A)]. This also allowed for normalized emittances to be extracted for $1 \sigma$ and $2 \sigma$ confidence bands. By including output from a previously developed computer program [21], the protons' momentums were adjusted for the energy degradation as they pass through each layer of $\mathrm{Cu}$ and film while recording the deposited energy. The deposited energy [Fig. 7(B)] was then weighted by an optical density curve [14] allowing direct comparison [Fig. 7(C)] of the simulated film and the experimental film.

\section{RESULTS AND DISCUSSION}

The simulated films seen in Fig. 8 are well in agreement with the experimental film. The focal spot diameter of the 6.7 $\mathrm{MeV}$ protons with the $8.5 \mathrm{~T}$ field is less than $2 \mathrm{~mm}$ (FWHM), and using the PHELIX spectrum from Fig. 3 and including the shot-to-shot error in proton numbers of at most $50 \%$, simulation indicates that the focal spot contains between $5.4 \times 10^{9}$ and $1.6 \times 10^{10}$ protons for $6.7 \pm 0.1 \mathrm{MeV}$. The phase-space plot [Fig. 9(A)] corresponding to this film layer shows the postsolenoid divergences of the laser accelerated protons. The Bragg peak at

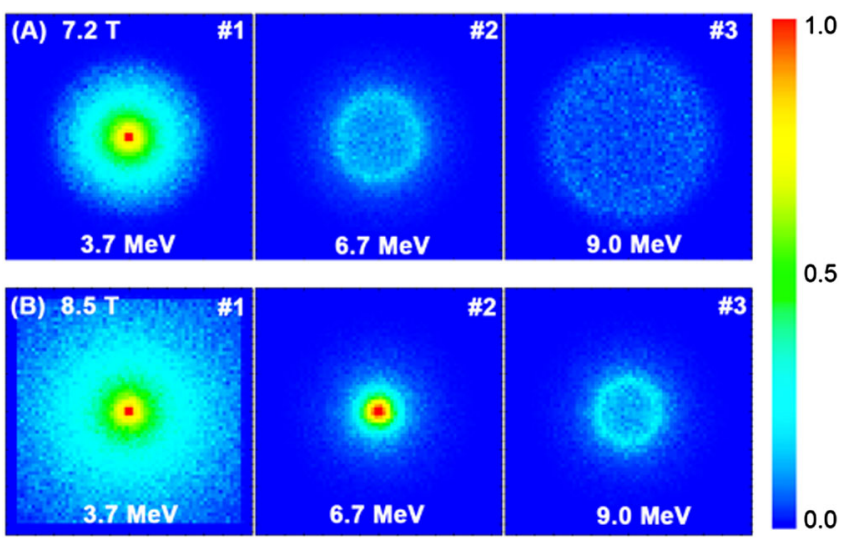

FIG. 8. Simulated film response from proton transport through fields of (A) $7.2 \mathrm{~T}$ and (B) $8.5 \mathrm{~T}$ for the first three films in each stack.

this layer corresponds to $6.7 \mathrm{MeV}$, and the $6.7 \mathrm{MeV}$ protons in the phase-space plot are highlighted in green. Figure 9 (B) is a projection onto the $x$ axis of the phase-space plot of (A), integrated over $\Theta$, and takes into account the exponentially decaying spectrum. The resulting narrow peak shows that most protons are concentrated on or near the axis at the position of the film stack, well in agreement with experimental results.

The phase-space plot in Fig. 10 for all laser accelerated protons that exit the solenoid further illustrates the severity of the chromatic aberration. The points highlighted in green represent $13.75 \pm 0.1 \mathrm{MeV}$ protons that are collimated by the $8.5 \mathrm{~T}$ field. The curved ends of the phasespace plot are a result of spherical aberration and are directly related to the limb brightening seen in Figs. 6 and 8. The area of an ellipse fit around the phase-space points is by definition the emittance, and if the curved ends of the collimated points were reduced, then the area and emittance are also reduced. Future solenoid designs may generate a more uniform magnetic field by increasing the number of windings at the ends compared to the center, increasing the radii of the middle windings compared to the outer windings, or some combination of both.
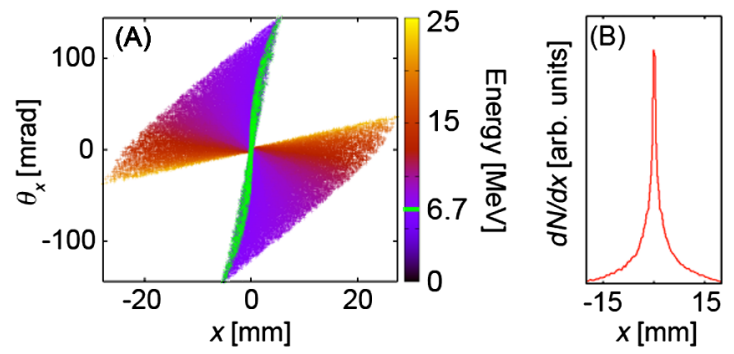

FIG. 9. (A) Phase-space plot for all protons at or above 6.7 MeV for $8.5 \mathrm{~T}$ (i.e. protons incident on film layer \#2 in Fig. 8). The green points highlight the $6.7 \mathrm{MeV}$ protons. (B) A projection of the phase-space plot onto the $x$ axis illustrates the proton density across the focal spot. 

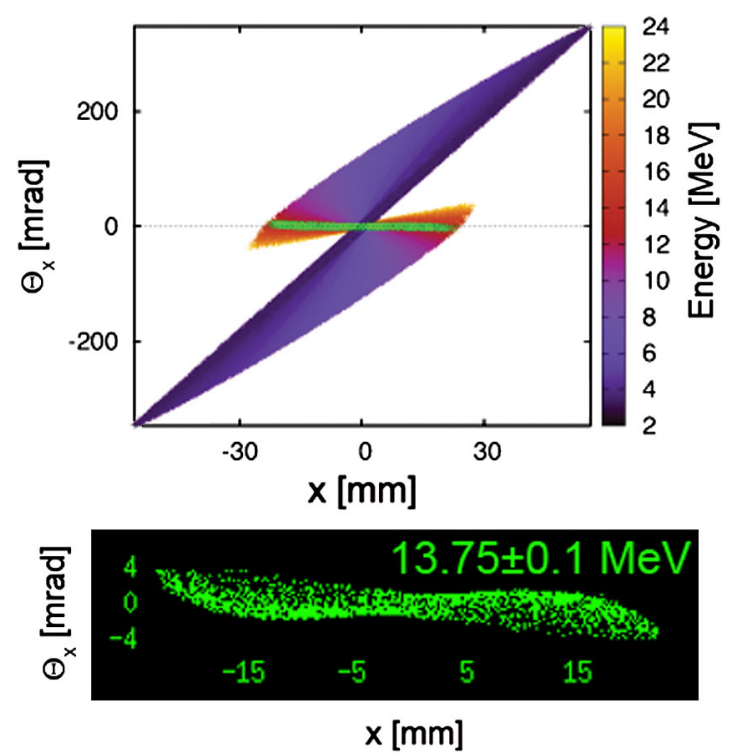

FIG. 10. A phase-space plot for all laser accelerated protons passing through the $8.5 \mathrm{~T}$ field. The green points (enlarged in lower image) highlight the $13.75 \pm 0.1 \mathrm{MeV}$ protons. The chromatic aberration results in divergent beams (yellow points), collimated beams (green points), focused beams (light-purple points), and beams that diverge after a focus (dark-purple points) at the film detector.

The inhomogeneous structures seen in the experimental film layers are absent from the simulation results, because the solenoid is modeled with concentric current loops and, therefore, has an azimuthally symmetric magnetic field for each point on the longitudinal axis. The structures are reproducible and only present after the proton beam passes through the magnetic field of the solenoid, suggesting that the inhomogeneities are not a result of the proton source, which can sometimes be the case for inhomogeneous laser focal spots [22]. Instead, they are a result of inhomogeneities in the magnetic field. The solenoid consists of four layers of wound wire. At the ends of the solenoid, the wire must be guided from one layer to the next while reversing direction, resulting in magnetic field inhomogeneities near the entrance and exit. The effects are induced in simulation by adding small perturbations to the magnetic field via offset longitudinal line currents positioned around each layer of the solenoid (Fig. 11).

The capture efficiencies of the presented solenoid coupled to the PHELIX laser using a $95 \mathrm{~mm}$ focal length are shown in Fig. 12. The 8.5 T field of our setup collimated $13.75 \mathrm{MeV}$ protons with near collimation of $13.75 \pm$ $1.25 \mathrm{MeV}$ protons. The laser accelerated protons are emitted from the source within a relatively uniform angular distribution, and most of the protons diverge off the axis of the solenoid. The number of protons passing through the solenoid on axis is 2 orders of magnitude less than the total transported spectrum. Therefore, their effect on transport efficiency calculations is negligible. For the $8.5 \mathrm{~T}$ magnetic field, $23 \%$ of the $13.75 \mathrm{MeV}$ laser accelerated protons are

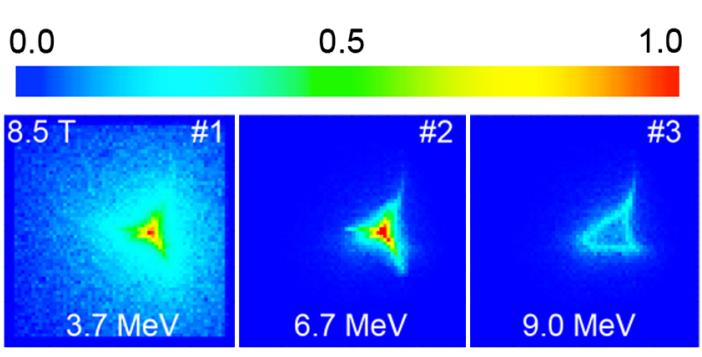

FIG. 11. Simulation of particle densities within dosimetric film layers illustrating the effect of higher order aberrations in the magnetic field.

captured and made parallel. The transport efficiencies of the lower energetic protons reach $34 \%$, and the $8.5 \mathrm{~T}$ magnetic field causes these protons to be focused before they continue to diverge away. Because the divergence angle at the source is smaller for higher energy laser accelerated protons (see Fig. 2), the transport efficiency of the protons on the high energy side of a spectrum is large, but these protons never reach a focus and are never collimated. Their divergence angles are only reduced.

For both accelerator injector applications and possible therapy applications, it becomes important to understand how many protons emerge from the solenoid and what their associated divergence angles are. One can see the number of protons emerging from the solenoid in Fig. 13 and that the spectra within the lower angular envelopes approach that of a quasimonoenergetic proton beam. There are $1.7 \times 10^{9}$ laser accelerated protons with divergence angles of $1 \mathrm{mrad}$ or less. This illustrates the solenoid's ability to act as an energy selector. This energy spread is important
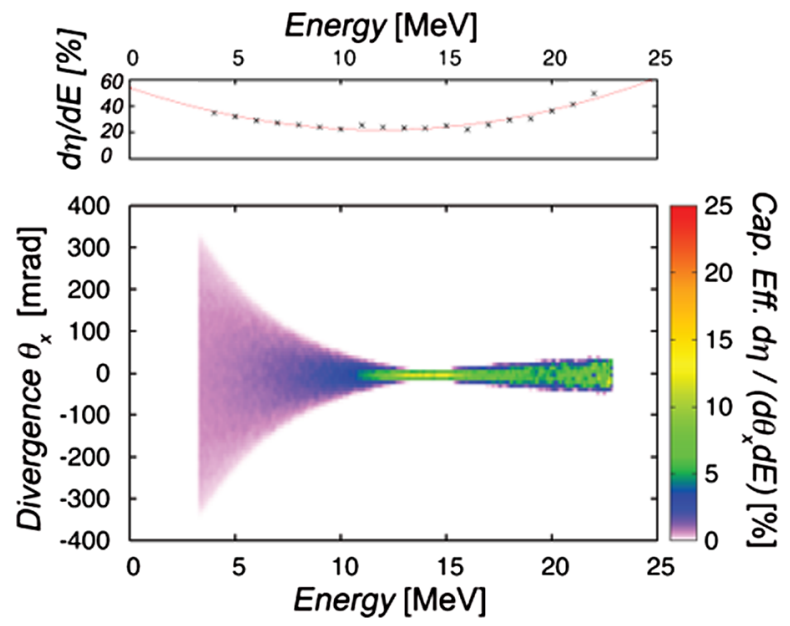

FIG. 12. Divergence angles $\Theta_{x}$ of protons after passing through the $8.5 \mathrm{~T}$ field of the solenoid. The color density indicates the transport efficiency $d \eta /\left(d \Theta_{x} d E\right)$ for a single energy and single angle while the top is a projection showing the total transport efficiency $d \eta / d E$ for a single energy across all angles. As governed by the $8.5 \mathrm{~T}$ field, $13.75 \mathrm{MeV}$ protons are collimated with near collimation of the $13.75 \pm 1.25 \mathrm{MeV}$ protons. 


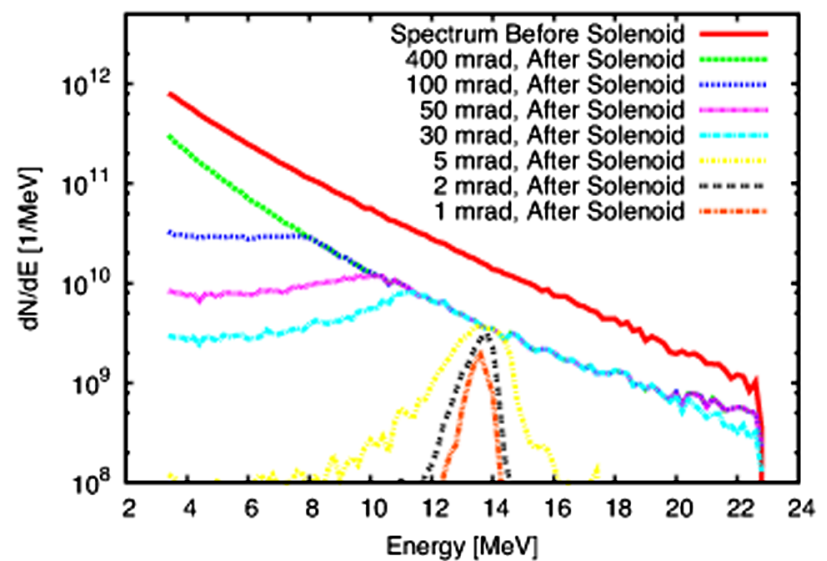

FIG. 13. Proton spectra within various angular envelopes after the $8.5 \mathrm{~T}$ solenoid field. The $1 \sigma$ and $2 \sigma$ confidence band normalized emittances for the protons within $1 \mathrm{mrad}$ are 2.5 and $10.2 \pi \mathrm{mm}$ mrad.

when coupling the laser accelerated proton source to a postaccelerator $\mathrm{rf}$ cavity, because the rf cavity will require a narrow energy spread. On the other hand, a typical requirement for proton therapy is a 2 Gy dose to a $1 \mathrm{~L}$ volume in a few minutes or less [23]. These requirements along with the constraints of available proton numbers and limited laser repetition rates suggest the need for a broad energy spectrum and the ability to perform a single-shot spread-out Bragg-peak (SOBP) dose deposition routine.

However, before performing a feasibility study of dose deposition routines, one needs to understand the characteristics of the particles available at the exit port of the beam delivery system. We, therefore, studied the feasibility of beam transport through a pulse power gantry, and offer a simulated concept (Fig. 14). We made two principle

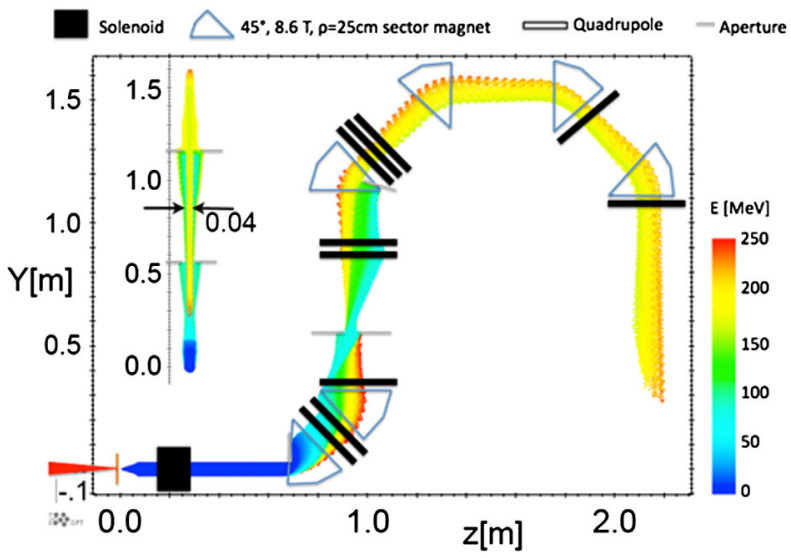

FIG. 14. Simulation results from feasibility study: Proton tracking of the scaled TNSA spectrum of Fig. 3 through a pulse power solenoid coupled to a theoretical achromatic pulse power gantry. The large image shows the $\mathbf{z}[\mathrm{m}], \mathbf{y}[\mathrm{m}]$ profile while the inset in the top left shows the $\mathbf{x}[\mathrm{m}], \mathbf{y}[\mathrm{m}]$ profile. The quadrupole gradients are up to $400 \mathrm{~T} / \mathrm{m}$ over $5 \mathrm{~cm}$ or less, and the dipole fields are $8.6 \mathrm{~T}$.

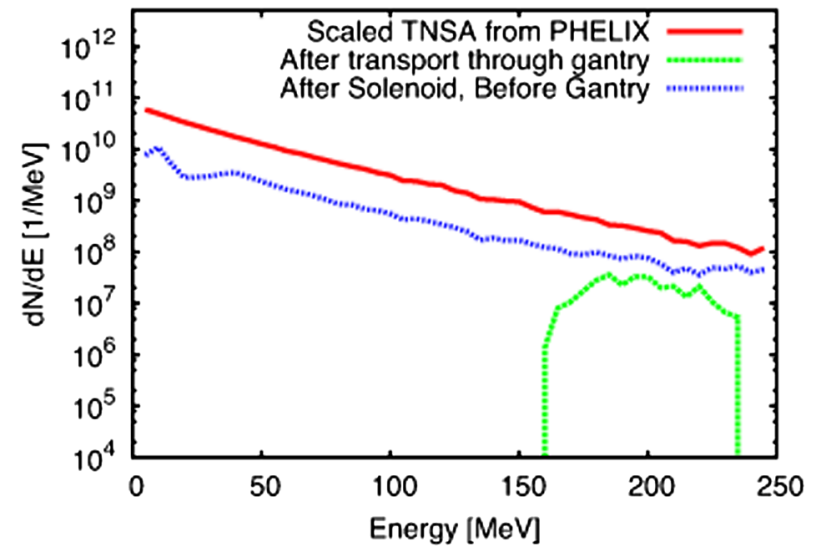

FIG. 15. Simulation of the proton spectrum after capture and collimation with a $32 \mathrm{~T}$ solenoidal field (blue, dotted curve) and transport through the simulated gantry presented in Fig. 14 (green, solid curve). When the solenoid field is set to zero, the spectrum emerging through the gantry is below $10^{4}$ protons per $\mathrm{MeV}$.

assumptions in this study. First, we assume a scaled TNSA spectrum with a maximum proton energy of $250 \mathrm{MeV}$ and the currently achievable $2 \times 10^{12}$ protons per pulse (Fig. 3, red dashed line). Second, we assume that we can develop sufficiently high quality air-core pulse quadrupoles (up to $400 \mathrm{~T} / \mathrm{m}$ over $5 \mathrm{~cm}$ ) and pulse dipoles that can be pulsed with the laser repetition rate. Additionally, the solenoid remains as the initial capture and collimating element, and its field was scaled to $32 \mathrm{~T}$. The spectrum after transport through the gantry is presented in Fig. 15 along with the scaled TNSA spectrum and the spectrum available after the solenoid. The broad spectrum after transport through the gantry indicates that a single-shot SOBP dose deposition routine may be possible.

Many technical challenges await laser acceleration, beam transport, and dose deposition handling, but for now we have demonstrated the highly efficient capture and transport of largely diverging laser accelerated protons and control over their large energy range with a first design pulse power solenoid. The magnetic field scales as $B \propto E_{p}{ }^{1 / 2}$, and present technology exists to collimate high energy laser accelerated protons. Alternative targetshaping concepts may reduce the beam divergence of TNSA and, therefore, enhance the performance of the solenoid [24]. Since the magnetic field strength of the solenoid is proportional to the current, some limiting factors when reaching higher magnetic field strengths may be mechanical stress [25] and temperature control [26]. However, the present outlook on pulse power technology looks promising, and one may even consider coupling a pulse power gantry to a conventional accelerator.

\section{ACKNOWLEDGMENTS}

We wish to thank the Phelix laser team and the coil fabrication group of the Dresden High Magnetic Field 
Laboratory for their expert support. We acknowledge M. Baumann, O. Boine-Frankenheim, W. Enghardt, F. Fiedler, I. Hoffmann, J. Pawelke, R. Sauerbrey, U. Schramm, and A. Wagner for useful conversations. We also acknowledge financial support by BMBF 06DA9044I and HIC4FAIR.

[1] R. A. Snavely et al., Phys. Rev. Lett. 85, 2945 (2000).

[2] T. Cowan et al., Phys. Rev. Lett. 92, 204801 (2004).

[3] M. Roth et al., Plasma Phys. Controlled Fusion 51, 124039 (2009).

[4] K. Harres et al., Phys. Plasmas 17, 023107 (2010).

[5] P. Antici, M. Fazi, A. Lombardi, M. Migliorati, L. Palumbo, P. Audebert, and J. Fuchs, IEEE Trans. Plasma Sci. 36, 1843 (2008).

[6] M. Nishiuchi et al., Phys. Rev. ST Accel. Beams 13, 071304 (2010).

[7] E. Fourkal, B. Shahine, M. Ding, J. Li, T. Tajima, and C. Ma, Med. Phys. 29, 2788 (2002).

[8] K. Ledingham, W. Galster, and R. Sauerbrey, Br. J. Radiol. 80, 855 (2007).

[9] H. Sakaki, M. Nishiuchi, T. Hori, P. Bolton, M. Tampo, A. Yogo, K. Kondo, S. Kawanishi, H. Iwase, and K. Niita, Plasma Fusion Res. 5, 9 (2010).

[10] T. Eichner, F. Grüner, S. Becker, M. Fuchs, D. Habs, R. Weingartner, U. Schramm, H. Backe, P. Kunz, and W. Lauth, Phys. Rev. ST Accel. Beams 10, 082401 (2007).

[11] M. Schollmeier et al., Phys. Rev. Lett. 101, 055004 (2008).

[12] M. Nishiuchi et al., Appl. Phys. Lett. 94, 061107 (2009).

[13] GafChromic HD-810 RadioChromic Film; International Specialty Products Corporation, Wayne, New Jersey [http://www.ispcorp.com].
[14] F. Nürnberg et al., Rev. Sci. Instrum. 80, 033301 (2009).

[15] Pulsar Physics, Eindhoven, The Netherlands [http:// www.pulsar.nl].

[16] V. Bagnoud et al., Appl. Phys. B 100, 137 (2009).

[17] H. Mercado-Uribe, I. Gamboa-deBuen, A. Buenfil, O. Avila, and M. Brandan, Nucl. Instrum. Methods Phys. Res., Sect. B 267, 1849 (2009).

[18] A. Niroomand-Rad, C. Blackwell, B. Coursey, K. Gall, J. Galvin, W. McLaughlin, A. Meigooni, R. Nath, J. Rodgers, and C. Soares, Med. Phys. 25, 2093 (1998).

[19] M. Abramowitz and I. A. Stegun, Handbook of Mathematical Functions (National Bureau of Standards, Washington, D.C., 1964).

[20] F. Nürnberg, A. Friedman, D. Grote, K. Harres, B. Logan, M. Schollmeier, and M. Roth, J. Phys. Conf. Ser. 244, 022052 (2010).

[21] M. Schollmeier, F. Nürnberg, and K. A. Flippo, Energy Loss In RadioChromic Film Matlab Script.

[22] J. Fuchs et al., Phys. Rev. Lett. 91, 255002 (2003).

[23] T. E. Cowan, U. Schramm, T. Burris-Mog, F. Fiedler, S. D. Kraft, K. Zeil, M. Baumann, M. Bussmann, W. Enghardt, K. Flippo, S. Gaillard, K. Harres, T. Herrmannsdoerfer, T. Kluge, F. Nurnberg, J. Pawelke, M. Roth, B. Schmidt, M. Sobiella, and R. Sauerbrey, AIP Conf. Proc. 1299, 721 (2010).

[24] P. Patel, A. Mackinnon, M. Key, T. Cowan, M. Foord, M. Allen, D. Price, H. Ruhl, P. Springer, and R. Stephens, Phys. Rev. Lett. 91, 125004 (2003).

[25] S. Zherlitsyn, T. Herrmannsdorfer, B. Wustmann, and J. Wosnitza, IEEE Trans. Appl. Supercond. 20, 672 (2010).

[26] P. Frings, H. Witte, H. Jones, J. Béard, and T. Hermannsdoerfer, IEEE Trans. Appl. Supercond. 18, 612 (2008). 